\title{
Broadband Electron Paramagnetic Resonance of a molecular spin triangle
}

Jérôme Robert, ${ }^{\mathrm{a}}$ Philippe Turek, ${ }^{\mathrm{b}}$ Matthieu Bailleul, ${ }^{\mathrm{a}}$ Athanassios K. Boudalis*abc

${ }^{a}$ Université de Strasbourg, CNRS, Institut de Physique et Chimie des Matériaux de Strasbourg (IPCMS), UMR 7504, F-67000 Strasbourg, France.

${ }^{b}$ Institut de Chimie de Strasbourg (UMR 7177, CNRS-Unistra), Université de Strasbourg, 4 rue Blaise Pascal, CS 90032, F-67081 Strasbourg, France.

${ }^{c}$ Institut de Science et d'Ingénierie Suparamolaiculaires - ISIS, 8 allée Gaspard Monge, BP 70028, F-67083 Strasbourg Cedex.E-mail: bountalis@unistra.fr.

\begin{abstract}
We built a broadband Electron Paramagnetic Resonance (EPR) spectrometer capable of fieldand frequency sweep experiments under field-, microwave amplitude- and microwave frequencymodulation detection modes (HM, AM, and FM, respectively). The spectrometer is based on a coplanar waveguide (CPW) architecture, with the sample being deposited on top of the transmission line. We tested the functionality of this spectrometer by measuring a standard 2,2-diphenyl-1-(2,4,6trinitrophenyl)hydrazyl (DPPH) sample, and complex $\left(\mathrm{N}^{n} \mathrm{Bu}_{4}\right)_{2}\left[\mathrm{Cu}_{3}\left(\mu_{3}-\mathrm{Cl}\right)_{2}(\mu-\mathrm{pz})_{3} \mathrm{Cl}_{3}\right]$ (1), dropcasted on the CPW. Complex 1 had been previously studied by conventional X-band EPR spectroscopy (Chem. Eur. J. 2020, 26, 12769-1784) and comparison with the past studies, validated the functionality of the spectrometer and confirmed the stability of the sample upon deposition. Moreover, our results highlighted the importance of surface effects and of the orientation of the microwave magnetic component $\mathbf{B}_{1}$ on the lineshapes of the recorded spectra.
\end{abstract}




\section{Introduction}

Classical EPR spectroscopy has been developed as a field-swept technique, using fixed frequencies. The reason is that the use of microwave cavities offers a huge increase in sensitivity due to their high quality factor (up to $10^{4}$ ), but these cavities can only function at specific wavelengths defined by their dimensions. However, variable-frequency magnetic resonance experiments are also of interest, as they can address systems with high zero-field splittings, and can overcome the problem of level anticrossings by selecting an appropriately strong magnetic field. Such experiments have indeed been demonstrated at drastically different frequency regimes.

In the THz range (far IR), magneto-infrared spectroscopy was invented in the 1960's by Richards $^{1-3}$ and used to this day to elucidate the magnetic properties of molecular magnetic materials, employing standard (Hg-lamp, globar) ${ }^{4}$ or synchrotron ${ }^{5}$ sources. Backward Wave Oscillator (BWO) sources are also used in quasioptical EPR set-ups, allowing one to achieve higher radiation intensities, though with the use of more cumbersome instrumentation. ${ }^{6}$ Such setups have limitations below $10 \mathrm{~cm}^{-1}(300 \mathrm{GHz})$ frequencies, necessitating the use of other techniques.

At the lower range of the $\mathrm{GHz}$ spectrum (up to $\sim 100 \mathrm{GHz}$ ), planar transmission lines, in particular coplanar waveguides (CPW), in conjunction with microwave signal generators, are widely used in broadband ferromagnetic resonance (FMR) in extended ferromagnets. ${ }^{7,8}$ The frequency range of these setups is of great interest in the study of molecular materials, however such use has been much less frequent due to the lower signals of such materials compared with ferromagnetic ones.

The EPR spectra of a few molecular magnetic materials have been measured using planar

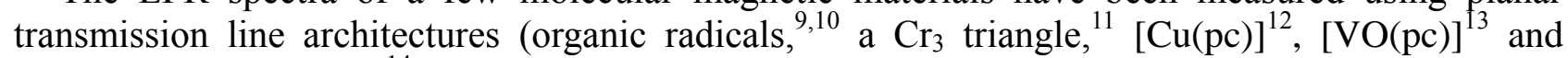
other metal complexes ${ }^{14}$ ). To our knowledge the only frequency domain study of molecular materials concerns the organic radicals 2,2-diphenyl-1-(2,4,6-trinitrophenyl)hydrazyl (DPPH) between 1-15 $\mathrm{GHz}^{15}$ and 2-(4'-p-methoxyphenyl)-4,4,5,5-tetramethylimidazoline-1-oxyl-3-oxide (NITPhOMe) carried out between 0.1-67 GHz. ${ }^{16}$

Most of these studies were conducted at low temperature, for which the relatively strong microwave absorption change could be resolved with the help of Vector Network Analyzer detection. Our interest in such materials prompted us to construct a broadband CPW-EPR spectrometer which uses various modulation schemes to increase its sensitivity, which we tested at room temperature using a previously reported spin triangle.

In particular, we demonstrate the utility of such a spectrometer by reporting the frequencydomain EPR spectroscopic study of the complex $\left(\mathrm{N}^{n} \mathrm{Bu}_{4}\right)_{2}\left[\mathrm{Cu}_{3}\left(\mu_{3}-\mathrm{Cl}\right)_{2}(\mu-\mathrm{pz})_{3} \mathrm{Cl}_{3}\right]$ (1, Figure 1) at room temperature, whose magnetic properties have been previously described in detail. ${ }^{17}$ Briefly, studies employing SQUID magnetometry, ab inito studies and continuous-wave (CW) X-band EPR spectroscopy on powders, solutions and single crystals, revealed that complex $\mathbf{1}$ exhibits intramolecular ferromagnetic interactions due to a poor overlap of the magnetic orbitals of the $\mathrm{Cu}^{\text {II }}$ ions. Moreover, its $S_{\mathrm{T}}=3 / 2$ ground state was shown to exhibit a moderate zero-field splitting (zfs), which partly derived from anisotropic exchange interactions, and partly from dipolar through-space interactions. As will be explained, these characteristics made it particularly adapted for this study (see below).

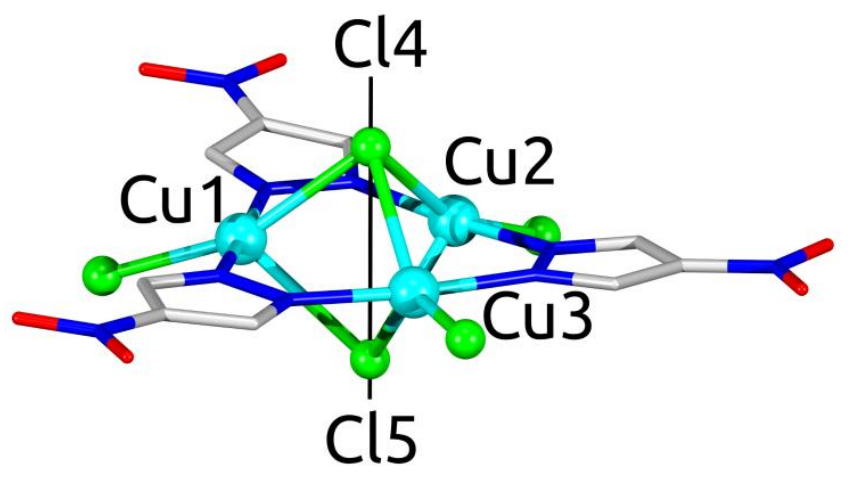


Figure 1. Partially labeled POV-Ray plot of the dianion of $\mathbf{1}$. Hydrogens are omitted for clarity. The line passing through $\mathrm{Cl} 4$ and $\mathrm{Cl} 5$ is normal to the $\mathrm{Cu}_{3}$ plane and defines the molecule's magnetic $z$ axis.

\section{Materials and methods}

Inspired by Montoya et al., ${ }^{18}$ we built a coplanar-waveguide-based EPR spectrometer which can be used in both fixed-frequency (FF, i.e. swept magnetic field) and frequency-sweep (FS, i.e. fixed magnetic field) modes. These are combined with 3 modulation techniques, namely standard magnetic field modulation (HM), frequency modulation (FM) and microwave amplitude modulation (AM). The external magnetic field range is up to $2 \mathrm{~T}$ and the frequency range is from 8 $\mathrm{MHz}$ to $50 \mathrm{GHz}$. Excitation power is usually $4 \mathrm{~mW}$. The basic components are shown in Figure 2.

The coplanar waveguide was fabricated using printed circuit board techniques on a $127 \mu \mathrm{m}$ thick PTFE/glass Rogers RT5880 substrate. It consists of a $300 \mu \mathrm{m}$ wide signal line, separated from the lateral ground planes by a $100 \mu \mathrm{m}$ gap. To ensure $50 \Omega$ single-mode operation up to $50 \mathrm{GHz}$, we adopted the channelized coplanar waveguide design: two dense rows of metallized vias connect these lateral ground planes to a bottom one, thus forming electrical walls preventing the excitation of higher order substrate modes. ${ }^{19}$ The $12.7 \times 10 \mathrm{~mm}^{2}$ S-shaped coplanar waveguide board is placed in a microwave box equipped with a pair of $2.4 \mathrm{~mm}$ non-magnetic Southwest connectors, and placed at the center of an electromagnet. The signal from the microwave generator to the CPW and the signal from the CPW to the Schottky diode are transmitted through $2.2 \mathrm{~mm}$ standard rigid coaxial cables. Such design has been used successfully for broadband ferromagnetic measurements. $8,20,21$

\section{Microwave generation and detection}

An Anritsu MG3695C signal generator is used to produce a continuous-wave microwave signal with a frequency between $8 \mathrm{MHz}$ and $50 \mathrm{GHz}$. The microwave signal transmitted through the CPW is rectified by a Herotek DZR50024 zero-bias Schottky diode. With an excitation power of $1 \mathrm{~mW}$, the voltage detected is several tenth of microvolts. The voltage output of the diode is fed to the input of a Stanford Research 860 lock-in amplifier.

\section{Sweep modes}

Fixed frequency (magnetic field sweep). External field sweeps are accomplished by varying the current in a Drusch electromagnet with a home-made power supply (Figure 2). controlled by the Aux port of the lock-in amplifier. The magnetic field is monitored by a Lakeshore 450 gaussmeter with GPIB488 acquisition interface.

Frequency-sweep (fixed magnetic field). The frequency of the signal generator is controlled directly through the GPIB488 interface, with an increment chosen in the acquisition software. In this mode, the constant external magnetic field is set manually on the power supply. The advantage of this mode is the quickness of acquisition compared to field sweep.

\section{Detection modes}

Various lock-in detection modes are available with the set up: field modulation, frequency modulation and amplitude modulation.

Field modulation (HM). This acquisition mode mimics standard commercial EPR spectrometers. Magneticfield modulation in the $0.01-2 \mathrm{mT}$ range is achieved using 2 coils taken from an old Varian X band EPR cavity. Each coil has an $8 \mathrm{ohm} \mathrm{DC} \mathrm{resistance} \mathrm{and} \mathrm{a} 1.2 \mathrm{mH}$ inductance (measured at $1 \mathrm{kHz}$ ). The sinusoidal modulation signal is provided by the reference output of the lock-in amplifier (frequency $105 \mathrm{~Hz}$, amplitude $0.6 \mathrm{~V}$ ), further amplified by a Samson audio power amplifier. Typical rms current inside a coil is about $2 \mathrm{~A}$.

Frequency modulation (FM). Reference signal $(100 \mathrm{~Hz}-10 \mathrm{kHz})$ from the lock-in amplifier is plugged in the $\mathrm{FM} / \phi \mathrm{M}$ input of the microwave generator. The amplitude of the reference is adjusted to obtain $5 \mathrm{MHz}$ of modulation. 
Amplitude modulation (AM). The TTL synchronisation signal (10 kHz) from the lock-in amplifier is applied to the Pulse trig in port of the microwave generator, thus providing a $100 \%$ amplitude modulation. In this mode, the signal detected by the Schottky diode represents directly the absorption, in contrast with the two modes described above where the signal detected represents the derivative of the absorption.

\section{Control}

The different instruments, namely the Stanford Research 860 lock-in amplifier, the Anritsu MG3695C microwave signal generator and the Lakeshore 450 gaussmeter are controlled with Labview through GPIB488 interface.

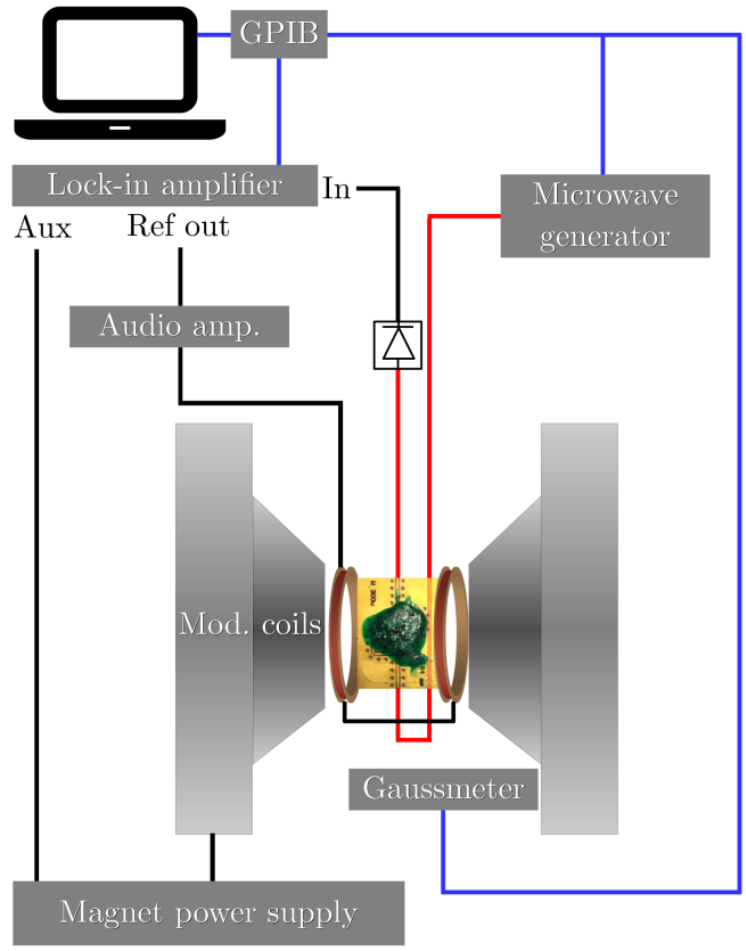

Figure 2. Schematics of the experiment. The molecular material is deposited on the coplanar wave guide (CPW) fed by the microwave generator (Anritsu MG3695C, $8 \mathrm{MHz}-50 \mathrm{GHz}, 4 \mathrm{~mW}$ ). The transmitted signal is rectified by a zero-bias Schottky diode (Herotek DZR50024) and amplified by the lock-in amplifier (Standford Research SR860). External magnetic field is applied by a Drusch electromagnet. Field modulation coils are powered through an audio power amplifier (Samson) with the reference signal of the lock-in amplifier. AM and FM modulations are described in the text and are not represented. The sample position is illustrated by an enlarged photo of the CPW.

\section{Sample}

For our study we selected the molecular complex salt $\left(\mathrm{N}^{n} \mathrm{Bu}_{4}\right)_{2}\left[\mathrm{Cu}_{3}\left(\mu_{3}-\mathrm{Cl}\right)_{2}(\mu-\mathrm{pz})_{3} \mathrm{Cl}_{3}\right](\mathbf{1}$, Figure 1), based on several criteria: (a) We had previously undertaken a thorough EPR study of this complex. ${ }^{17}$ (b) These studies revealed that its $S=3 / 2$ ground state is characterized by a modest zerofield splitting $\left(\sim 0.1 \mathrm{~cm}^{-1}\right.$, which corresponds to $\left.3 \mathrm{GHz}\right)$, ideal for study within the $0-50 \mathrm{GHz}$ range accessible by our spectrometer. (c) This zfs gives rise to EPR signals covering a broad frequency and/or magnetic field range. Thus, this material can be used for the validation of our spectrometer's operation over a broader set of experimental conditions that those offered by simple organic radicals with narrow signals.

Approximately $0.1 \mathrm{~g}$ of crystallites of 1 were dissolved in dichloromethane $(1 \mathrm{~mL})$ and 10 droplets of the solution (ca. $0.5 \mathrm{~mL}$ ) were evaporated on the CPW surface, amounting to ca. $50 \mathrm{mg}$ of sample (Figure 3). For that quantity, a conversion time of $2 \mathrm{~ms}$ and 500 averages per point were used, leading to reasonable spectral accumulation times.

The same CPW sample holder could be easily used to measure different samples, after thorough rinsing with the appropriate solvents $\left(\mathrm{CH}_{2} \mathrm{Cl}_{2}\right.$ in this case) and drying under a nitrogen stream. 


\section{Fitting}

Spectra were fitted using Easyspin v. 6.0, ${ }^{22}$ employing home-made Matlab routines. Depending on the experiment, spectra were fitted either individually, or simultaneously using a common set of parameters using Easyspin's pepper function. Line widths, $\sigma$, are calculated in $\mathrm{mT}$ and $\mathrm{MHz}$ for field-and frequency-swept experiments, respectively. In our fits, simple convolutional broadening was considered, in which case the conversion is $\sigma_{H}(\mathrm{mT})=\sigma_{f}(\mathrm{MHz}) \cdot h \cdot 10^{9} / g \mu_{B}{ }^{23}$ To account for the different $\mathbf{B}_{1}$ orientations of the experiment inside the $\mathbf{B}_{0}$ field, a rough approximation was tested, whereby parallel- and perpendicular mode spectra were calculated, with each contribution being considered in the final spectrum according to a weighing parameter (indicative simulations are shown in Figures S1 and S2). However, no such contribution was revealed by any of the fits, so these attempts are omitted for brevity.

Moreover, due to the sample being surface-deposited through drop casting of a dilute solution, we considered the possibility that it undergoes ordering along some preferential orientation relative to the CPW surface. This was modeled through Easyspin's ordering function $P(\theta)=\mathrm{e}^{-U(\theta)}$ with $U(\theta)$ $=-\lambda\left(3 \cos ^{2} \theta-1\right) / 2 ; \lambda<0$ implies preferential orientation in the molecule's magnetic $x y$-plane, and $\lambda>0$ along the molecule's magnetic $z$-axis.

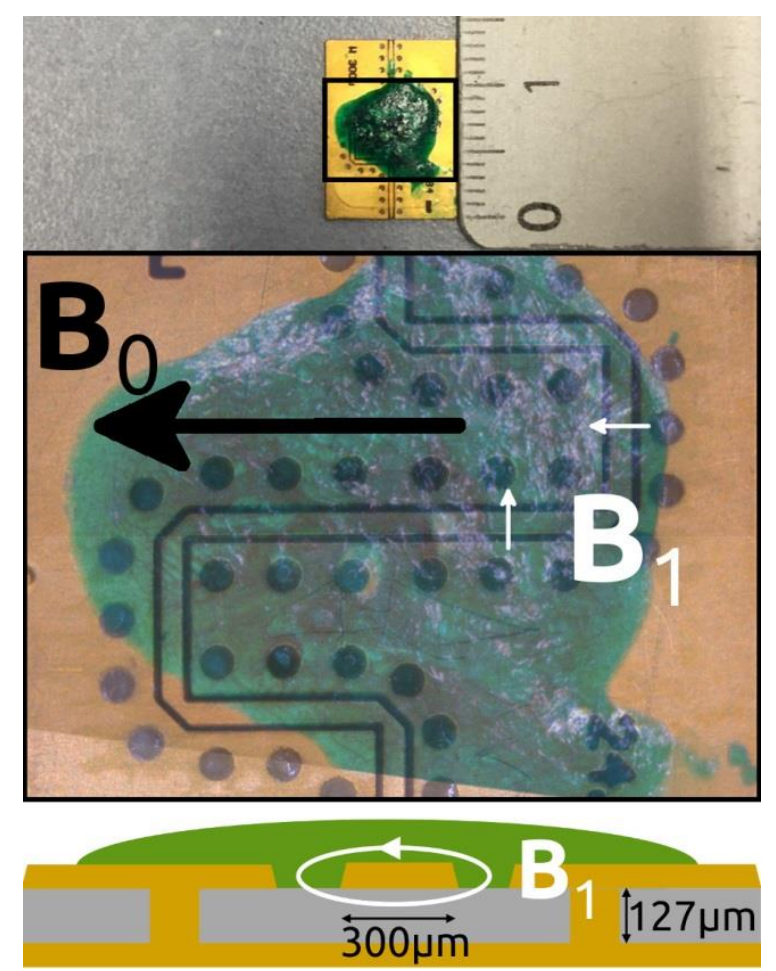

Figure 3. Top. Photo of the evaporated sample on the coplanar waveguide. Middle. Composite enlargement showing the transmission line below the evaporated sample. The Zeeman $\left(\mathbf{B}_{0}\right)$ field is shown in black and the top projections of the excitation $\left(\mathbf{B}_{1}\right)$ magnetic field vectors are shown in white at different sections of the transmission line. Bottom. A cross section of the CPW showing the map of the $\mathbf{B}_{1}$ field and how it penetrates the evaporated sample (shown in green).

\section{Results}

Prior to testing complex 1, we carried out preliminary tests with the well-known radical DPPH in field-sweep mode. For comparison, spectra were collected at $17.000 \mathrm{GHz}$ both under HM and AM detection modes (Figure 4). Fits, considering $g=2.0037$ were used to calibrate for the magnetic field offset. These revealed a narrow, mostly Lorentzian, symmetric signal with Gaussian and Lorentzian line width components (FWHM) $\sigma_{\mathrm{G}}=0.082 \mathrm{mT}, \sigma_{\mathrm{L}}=0.56 \mathrm{mT}$ for the AM-detected spectrum and $\sigma_{\mathrm{G}}=0.068 \mathrm{mT}, \sigma_{\mathrm{L}}=0.38 \mathrm{mT}$ for the HM-detected spectrum. These fall in the range 
previously reported for DPPH, whose line width is known to heavily depend on the solvent of crystallization ${ }^{24}$ and to be broadened by the presence of dioxygen. ${ }^{25}$

These experiments demonstrated that the HM detection afforded a better $\mathrm{S} / \mathrm{N}$ ratio and was therefore used for subsequent experiments.

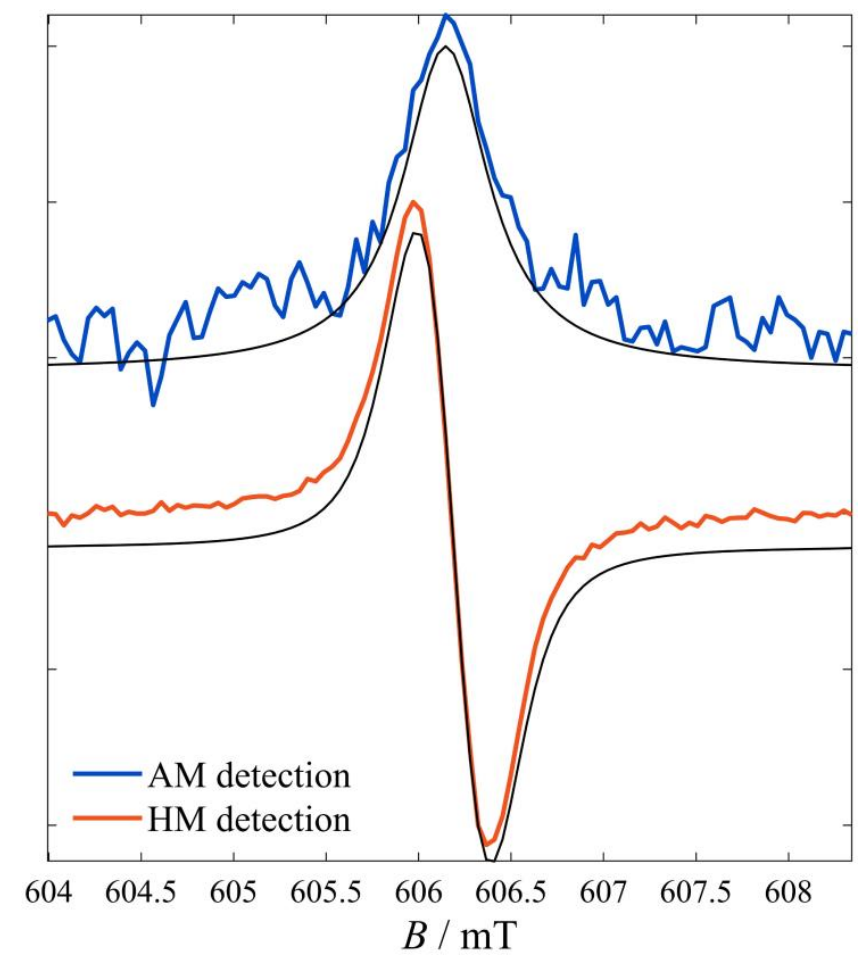

Figure 4. Room-temperature spectra of DPPH in AM and HM detection modes. The black lines are fits to single species. Experimental conditions. $f_{\mathrm{EPR}}=17.000 \mathrm{GHz}, P_{\mathrm{MW}}=4 \mathrm{~mW}$. AM: $\Delta P_{\bmod }=4$ $\mathrm{mW}(0-100 \%)$. HM: $\Delta B_{\text {mod }}=5 \mathrm{G}_{\mathrm{pp}}$.

We then proceeded to examine complex 1. For comparison to subsequent experiments, dual (Xand Q-band) frequency spectra of a powder of 1 were recorded in regular EPR cavities in fieldswept mode (Figure 5). Results were analysed using a simple spin Hamiltonian with zero-field splitting and Zeeman terms:

$$
\hat{H}_{3 / 2}=D \hat{S}_{z}^{2}+\mu_{B} \mathbf{H} \tilde{\mathbf{g}} \hat{\mathbf{s}}(1)
$$

where $S=3 / 2$ and only the axial zfs term, $D$ was considered. Our previous work on $\mathbf{1}$ and on related complexes has demonstrated that this is a valid approach, ${ }^{17}$ and EPR spectra of $\mathbf{1}$ are analysed according to this Hamiltonian throughout this work.

Simultaneous fits were in perfect agreement to the previously published results, with best-fit parameters: $g_{\|}=2.212, g_{\perp}=2.073, D=0.105 \mathrm{~cm}^{-1}$, with Gaussian and Lorentzian line width contributions of $\sigma_{\mathrm{G}}=16.37 \mathrm{mT}$ and $\sigma_{\mathrm{L}}=9.44 \mathrm{mT}$, respectively. 

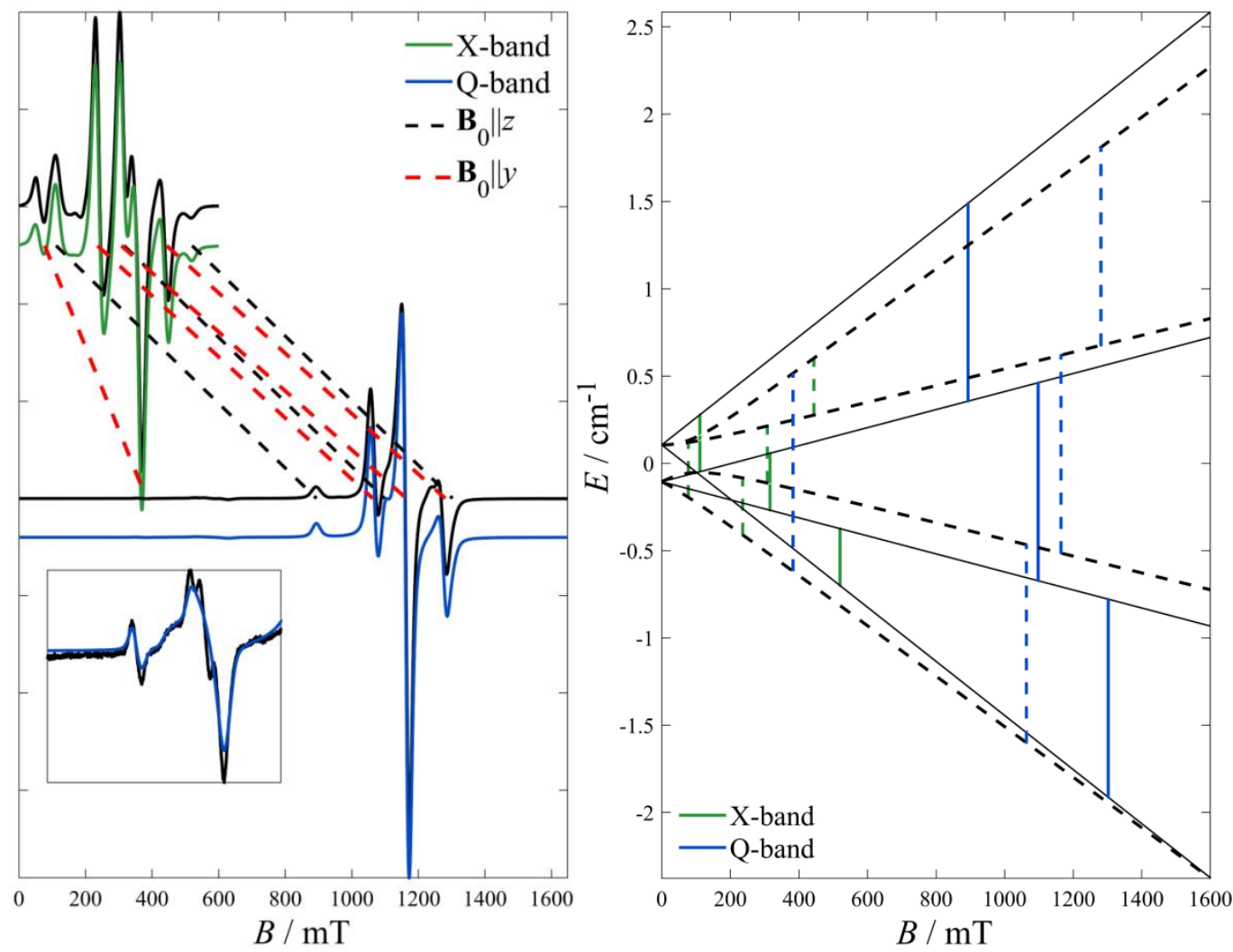

Figure 5. Left: Room-temperature X- and Q-band EPR spectra of 1 collected from regular EPR cavities (black lines) and simultaneous fits to a common parameter set (colored lines). The dashed straight lines indicate the shift of the resonance magnetic fields for the transitions at the $\mathbf{B}_{0} \| z$ and $\mathbf{B}_{0} \| y$ orientations. The inset shows an expansion of the Q-band spectrum (and fit) revealing the forbidden $\Delta M_{S}= \pm 3(\sim 400 \mathrm{mT})$ and $\Delta M_{S}= \pm 2(\sim 600 \mathrm{mT})$ transitions. The substructure in the 600 $\mathrm{mT}$ feature is attributed to partially resolved hyperfine interactions. Right: Zeeman plots of the system indicating the transitions for the best-fit solution at the two frequencies and at the $\mathbf{B}_{0} \| z$ (solid lines) and $\mathbf{B}_{0} \| y$ (dashed lines) orientations. The $\Delta M_{S}= \pm 2$ transitions at the Q-band are only observed under intermediate orientations. Experimental parameters. X-band: $f_{\mathrm{EPR}}=9.778 \mathrm{GHz}$, $P_{\mathrm{MW}}=1.95 \mathrm{~mW}, B_{\mathrm{mod}}=5 \mathrm{G}_{\mathrm{pp}}$. Q-band: $f_{\mathrm{EPR}}=34.004 \mathrm{GHz}, P_{\mathrm{MW}}=0.28 \mathrm{~mW}, \Delta B_{\mathrm{mod}}=5 \mathrm{G}_{\mathrm{pp}}$.

These spectra were compared to field-swept spectra collected on the CPW at comparable frequencies (9.514 and $38.056 \mathrm{GHz}$, respectively), shown in Figure 6. The X-band spectrum faithfully reproduced the overall characteristics of its cavity-collected counterpart, with best-fit parameters $g_{\|}=2.156, g_{\perp}=2.101, D_{1}=0.093 \mathrm{~cm}^{-1}, \sigma_{\mathrm{G}}=8.92 \mathrm{mT}, \sigma_{\mathrm{L}}=7.32 \mathrm{mT}$. These slight differences were explained by the effects of surface deposition (see below).

However, the Q-band spectrum exhibited more marked differences, in particular with the appearance of additional features indicating the presence of two components. Fits were therefore conducted with two different $S=3 / 2$ systems exhibiting zfs. Best-fit parameters were: $g_{1 \|}=2.254$, $g_{1 \perp}=2.077, D_{1}=0.120 \mathrm{~cm}^{-1}, \sigma_{D}=0.012 \mathrm{~cm}^{-1}, \sigma_{1 \mathrm{G}}=21.33 \mathrm{mT}, \sigma_{1 \mathrm{~L}}=2 \mathrm{mT}$ (fixed), $\lambda_{1}=0.5$ (fixed) for component 1 , and $g_{2 \|}=2.204, g_{2 \perp}=2.046, D_{2}=0.071 \mathrm{~cm}^{-1}, \sigma_{2 \mathrm{G}}=18.81 \mathrm{mT}, \sigma_{2 \mathrm{~L}}=2 \mathrm{mT}$ (fixed), $\lambda_{2}=1.5$ (fixed) for component 2 with a 1:0.3 relative weight ratio, respectively. 


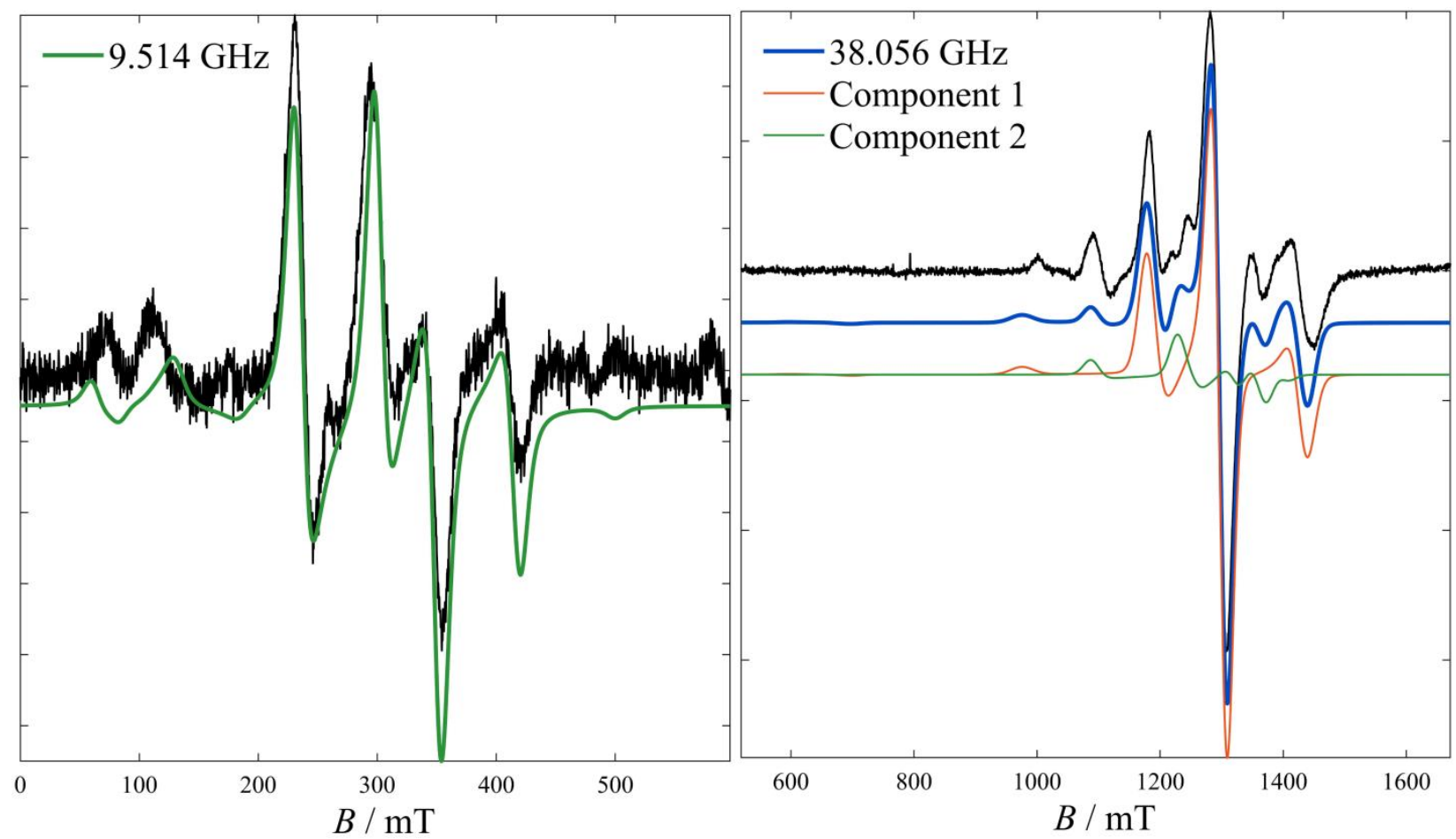

Figure 6. Room-temperature X- and Q-band EPR spectra of 1 collected from the CPW spectrometer (black lines). Fits are according the description in the text. Experimental parameters. $P_{\mathrm{MW}}=4$ $\mathrm{mW}, \Delta B_{\mathrm{mod}}=5 \mathrm{G}_{\mathrm{pp}}$. X-band: $f_{\mathrm{EPR}}=9.514 \mathrm{GHz}$. Q-band: $f_{\mathrm{EPR}}=38.056 \mathrm{GHz}$.

Multifrequency EPR spectra of 1 were recorded using HM detection and reported as first derivative spectra on a frequency (Figure 7) and on a $g$-scale (Figure S3). In modeling these spectra we employed the giant-spin approximation, considering a $S=3 / 2$ system experiencing zero-field splitting (zfs).

Field-swept and frequency-swept spectra of 1 reproduced the general features of the resonator spectra, revealing a complicated spectrum at low fields/frequencies, which is deconvoluted at higher fields/frequencies. However, these deconvoluted spectra exhibit more resonances than the Q-band spectrum reported above, pointing toward the presence of at least two components.

The presence of a second component can be rationalized making two considerations: (i) Different molecular orientations may be stabilized for on-surface deposited molecules with respect to quasi-bulk molecules in layers further from the CPW surface. (ii) This complex has been known to form at least four different polymorphs depending on the crystallization conditions, each with its own subtle structural differences. ${ }^{26}$ Since structural parameters have been found to modulate the precise magnitude of the ground state $\mathrm{zfs},{ }^{17}$ different $D$ values may reasonably explained by the presence of such polymorphs.

Frequency-swept spectra fitted based on the above considerations could be nicely reproduced assuming two components, with best-fit parameters $g_{1 \|}=2.243, g_{1 \perp}=2.074, D_{1}=0.119 \mathrm{~cm}^{-1}, \sigma_{1 \mathrm{G}}=$ $274 \mathrm{MHz}, \sigma_{1 \mathrm{~L}}=180 \mathrm{MHz}$, for component 1 , and $g_{2 \|}=2.217, g_{2 \perp}=2.113, D_{2}=0.091 \mathrm{~cm}^{-1}, \sigma_{2 \mathrm{G}}=$ $567 \mathrm{MHz}, \sigma_{2 \mathrm{~L}}=17 \mathrm{MHz}$, for component 2, with relative weights of 1:0.217, respectively.

According to this line shape modeling, this solution suggests that the majority of the sample exhibits spin Hamiltonian parameters similar to those determined in powder and in solution. At the same time, a minority component exhibits a slightly smaller zfs,. However, it must be understood that in this multivariate problem, such assignments have rather qualitative character.

Moreover, it should be noted that introduction of an ordering parameter to the fitting model yielded only marginal improvements to the fits of these experiments. We are currently investigating the suitability of various models to describe the line shapes of field-swept EPR spectra from surface-deposited samples. 


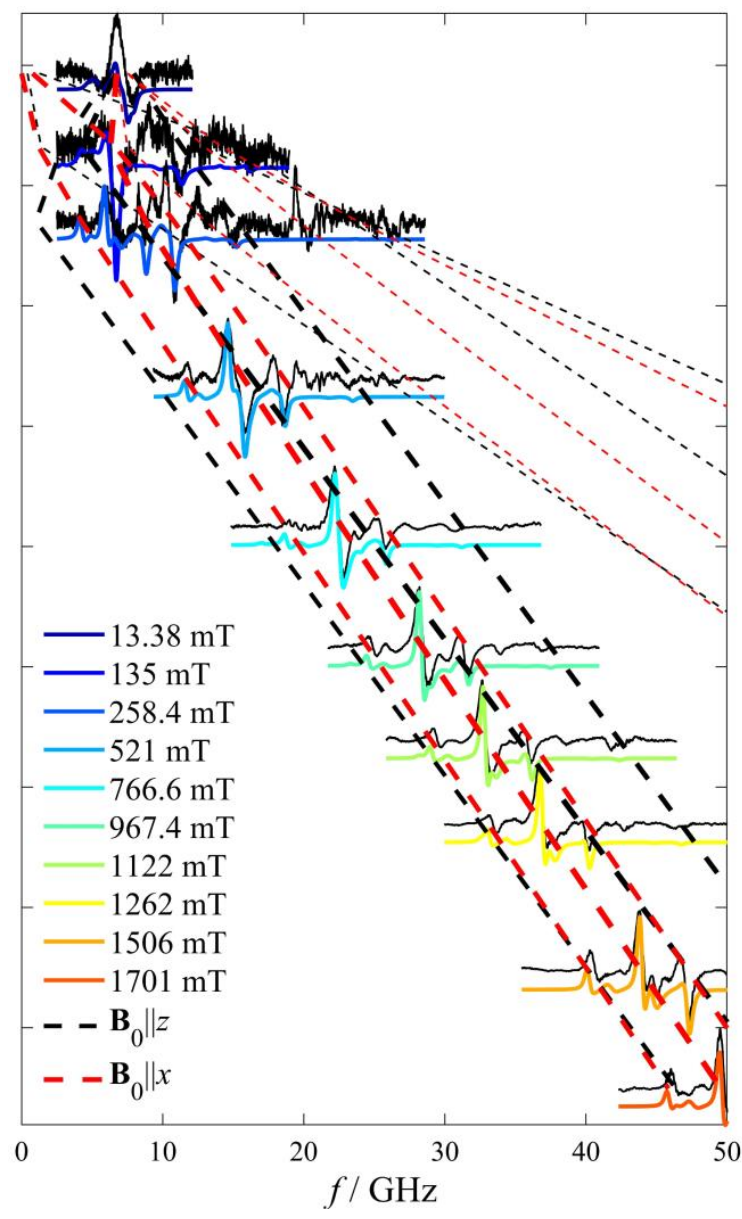

Figure 7. Room-temperature broadband EPR data for $\mathbf{1}$ at various magnetic fields (black lines) and fits according to the model described in the text (colored lines). The dashed lines indicate the resonance frequencies of selected transitions at two molecular orientations of component 1 . The line thicknesses are indicative of the transition probability amplitudes. Experimental conditions. $P_{\mathrm{MW}}$ $=4 \mathrm{~mW} . \Delta B_{\bmod }=5 \mathrm{G}_{\mathrm{pp}}$.

\section{Discussion and conclusions}

From a method development perspective, in this work we demonstrated the construction of a broadband EPR spectrometer based on the CPW architecture, and the implementation of several modulation detection schemes which provide increased sensitivity for the measurement of molecular magnetic materials.

These materials exhibit inherently weaker signals with respect to ferromagnets, which are typically studied with CPW architectures, and this design proved suitable for the measurement of their EPR spectra even at room temperature. Fits to these EPR spectra reproduced the spin Hamiltonian parameters previously determined from classical EPR spectroscopy, thus validating the functionality of this new spectrometer.

It should be noted that this design offers considerable flexibility for the EPR study of molecular magnetic materials, as it allows the arbitrary selection of microwave frequency within a very broad range, typically up to $100 \mathrm{GHz}$, depending on the available source. Thus, it allows the study of zfs systems with large anisotropies, usually not accessible to X-band setups.

Moreover, it offers the possibility to partially reproduce dual-mode experiments, typically carried out with dual-mode cavities: instead of selecting the cavity's parallel or perpendicular mode, rotation of the CPW will have a comparable effect. For a sample deposited only on the middle (horizontal) portion of the transmission line as shown in Figure 3, all $\mathbf{B}_{1}$ vectors are perpendicular to $\mathbf{B}_{0}$. Turning the CPW by $90^{\circ}$ does not entiely eliminate the perpendicular $\mathbf{B}_{1}$ component, since 
the magnetic lines are curved, but does introduce a parallel component. Comparison of the two spectra could allow the observation of the amplification of forbidden transitions.

Moreover the results from the EPR study of complex 1 justified our choice of this material: being a moderate-zfs system it presented several crossings and anticrossings of its levels, yielding characteristic features at low magnetic fields or frequencies. Not only were these features useful for assessing the stability of the complex upon deposition, but also in assessing eventual orientation effects, something not possible with highly isotropic radical signals.

Regarding the comparison between the frequency- and field-swept modes accessible with this setup, a clear advantage of the frequency-swept mode is that it simplifies the simulations of spectral line shapes and intensities. With respect to line intensities, the Aasa-Vänngård " $1 / g$ " correction is not required. ${ }^{27}$ With respect to line shapes, their analysis is based on the more fundamental frequency-domain line broadening, thus not requiring assumptions for the conversion to fielddomain line shapes. These latter may deviate from purely Gaussian/Lorentzian ones when the energy-level dependence on the magnetic field is nonlinear, ${ }^{23}$ and may be highly anisotropic for different $g$-ranges. Finally, looping transitions do not occur in frequency-swept mode, even if the selected magnetic field is at an anticrossing point. ${ }^{28}$

Moreover, from a purely instrumental perspective, and for the same experimental parameters, a frequency sweep can be much faster than a field sweep, since a frequency increment can be almost instantaneous, whereas a field increment is limited by the inductance of the coil which increases the settling time, and by the time needed to read the Hall probe. Thus, the frequency-swept mode can additionally enhance the $\mathrm{S} / \mathrm{N}$ ratio for given conversion times and number of averaging scans.

Regarding the sample form, one overall conclusion that has been drawn from these studies is that EPR spectroscopy of surface-deposited samples may not be an entirely trivial issue regarding (i) the stability of the deposited samples and (ii) the lineshape analysis of the spectra. Thus, while nondestructive deposition was accomplished in this case, special care should be taken to confirm the identity of less stable materials. Moreover, even in the case of samples deposited with retention of their structural integrity, attention will need to be given to orientational ordering effects on the surface as well as to the orientations of the $\mathbf{B}_{1}$ vectors inside $\mathbf{B}_{0}$ and the corresponding transition probability amplitudes.

In conclusion, a new field- and frequency-sweep broadband EPR spectrometer has been built and described, incorporating different detection schemes. Our preliminary studies have demonstrated its suitability in the study of molecular magnetic materials even at room temperature, thus making it an attractive option for systems requiring access to a broad range of excitation frequencies.

\section{Acknowledgments}

The authors thank the Fondation pour la Recherche en Chimie (Foundation for Frontier Research in Chemistry) for support through the "MiSSTri" project ("Microwave Spectroscopy of Spin Triangles", Innovation Grant PTu-FRC-0003). They also thank Prof. Raphael G. Raptis for the gracious supply of a sample of complex 1, José Solano for help with computer programming and Pascal Leindecker from the Heterogeneous Systems and Microsystems group of the Icube lab for help with the construction of parts of the spectrometer.

\section{References:}

1 P. L. Richards, Journal of the Optical Society of America, 1964, 54, 1474.

2 R. R. Joyce and P. L. Richards, Physical Review, 1969, 179, 375-380.

3 G. C. Brackett, P. L. Richards and W. S. Caughey, The Journal of Chemical Physics, 1971, 54, 4383-4401.

4 P. C. Bunting, M. Atanasov, E. Damgaard-Møller, M. Perfetti, I. Crassee, M. Orlita, J. Overgaard, J. van Slageren, F. Neese and J. R. Long, Science, 2018, 362, eaat7319.

5 A. Schnegg, J. Behrends, K. Lips, R. Bittl and K. Holldack, Phys. Chem. Chem. Phys., 2009, 11, 6820 . 
6 J. van Slageren, S. Vongtragool, B. Gorshunov, A. A. Mukhin, N. Karl, J. Krzystek, J. Telser, A. Müller, C. Sangregorio, D. Gatteschi and M. Dressel, Physical Chemistry Chemical Physics, 2003, 5, 3837-3843.

7 C. Bilzer, T. Devolder, P. Crozat, C. Chappert, S. Cardoso and P. P. Freitas, Journal of Applied Physics, 2007, 101, 074505.

8 J. M. Shaw, H. T. Nembach, T. J. Silva and C. T. Boone, Journal of Applied Physics, 2013, 114, 243906.

9 C. Bonizzoni, F. Troiani, A. Ghirri and M. Affronte, Journal of Applied Physics, 2018, 124, 194501.

10 A. Ghirri, C. Bonizzoni, F. Troiani, N. Buccheri, L. Beverina, A. Cassinese and M. Affronte, Physical Review A, , DOI:10.1103/PhysRevA.93.063855.

11 A. Ghirri, C. Bonizzoni, M. Righi, F. Fedele, G. Timco, R. Winpenny and M. Affronte, Applied Magnetic Resonance, 2015, 46, 749-756.

12C. Bonizzoni, A. Ghirri and M. Affronte, Advances in Physics: X, 2018, 3, 1435305.

13C. Bonizzoni, A. Ghirri, M. Atzori, L. Sorace, R. Sessoli and M. Affronte, Scientific Reports, , DOI:10.1038/s41598-017-13271-w.

14C. Bonizzoni, A. Ghirri, K. Bader, J. van Slageren, M. Perfetti, L. Sorace, Y. Lan, O. Fuhr, M. Ruben and M. Affronte, Dalton Transactions, 2016, 45, 16596-16603.

15 K. Jing, Z. Lan, Z. Shi, S. Mu, X. Qin, X. Rong and J. Du, Review of Scientific Instruments, $2019,90,125109$.

16Y. Wiemann, J. Simmendinger, C. Clauss, L. Bogani, D. Bothner, D. Koelle, R. Kleiner, M. Dressel and M. Scheffler, Applied Physics Letters, 2015, 106, 193505.

17L. Mathivathanan, G. Rogez, N. Ben Amor, V. Robert, R. G. Raptis and A. K. Boudalis, Chem. Eur. J., 2020, 26, 12769-12784.

18E. Montoya, T. McKinnon, A. Zamani, E. Girt and B. Heinrich, Journal of Magnetism and Magnetic Materials, 2014, 356, 12-20.

19R. Simons, Coplanar waveguide circuits, components, and systems, John Wiley, New York, 2001.

20 M. Bailleul, Applied Physics Letters, 2013, 103, 192405.

21 I. S. Camara, C. Achkar, N. Liakakos, A. Pierrot, V. Pierron-Bohnes, Y. Henry, K. Soulantica, M. Respaud, T. Blon and M. Bailleul, Applied Physics Letters, 2016, 109, 202406.

22 S. Stoll and A. Schweiger, Journal of Magnetic Resonance, 2006, 178, 42-55.

23 J. R. Pilbrow, Bulletin of Magnetic Resonance, 1988, 10, 32-64.

24 S. A. Al'tshuler and B. M. Kozyrev, Electron Paramagnetic Resonance., Academic Press Inc, London, 1964.

25 H. Inokuchi and M. Kinoshita, BCSJ, 1960, 33, 1627-1629.

26G. Mezei, University of Puerto Rico, 2004.

27R. Aasa and tore Vänngård, Journal of Magnetic Resonance (1969), 1975, 19, 308-315.

28 J. R. Pilbrow, G. R. Sinclair, D. R. Hutton and G. J. Troup, Journal of Magnetic Resonance (1969), 1983, 52, 386-399. 